\title{
Política y práctica social basada en la evidencia: ¿Una nueva ideología o un imperativo de Derechos Humanos?*
}

\section{Evidence based social policy and practice: A new ideology or a Human Rights imperative?}

\section{PhD. Ann Buchanan}

Ann Buchanan, PhD, M.A (Oxon), CQSW; es Directora del Oxford Centre for Research into Parenting and Children, además es profesora en Work at Bamett House y Fellow de St Hilda's College. Dirección postal: Department of Social Policy and Social Work, Barnett House, 32 Wellington Square, Oxford OX1 2ER, UK Dirección de e-mail: ann.buchanan@socres.ox.ac.uk

\begin{abstract}
Resumen
En este artículo se sostiene que el Trabajo Social y la política social basados en la evidencia son un imperativo de derechos humanos. Ya no es aceptable que al momento de formular políticas sociales los gobiernos o los agentes de Trabajo Social, como los trabajadores sociales, se involucren en la vida de las personas sin una evidencia que demuestre que lo que hacen puede ser beneficioso - o al menos no dañino- para quienes son el objetivo de la política o la intervención. Este estudio sostiene que cuando los profesionales y legisladores intervienen en la vida de los demás sin una evidencia que muestre que lo que hacen podría ser beneficioso para los involucrados, no son menos culpables del daño causado y de abuso de derechos humanos simplemente porque pretendian hacer el bien. Este trabajo se divide en dos partes. La primera considera el caso del Trabajo Social y sus implicancias para la enseñanza de Trabajo Social, y la segunda considera la política social.
\end{abstract}

Palabras clave. Trabajo Social, política social, práctica basad en la evidencia, derechos humanos.

\begin{abstract}
This paper argues that evidence based social work and social policy is a human rights imperative. It is no longer acceptable, for governments when formulating social policies or social work agents, such as social workers, to become involved in people's lives without some evidence that what they are doing may be beneficial or at the very least not harmful to those who are the target of the policy or intervention. This paper suggests where professionals and policy makers intervene in the lives of others without evidence that what they are doing is likely to be beneficial to the individuals involved, they are no less culpable of harm caused and human rights abuse simply because they intended to do good. This paper is in two parts. The first part considers the case of social work and it implications for the teaching of social work and the second part considers social policy.
\end{abstract}

Key works. Social work, social policy, evidence based practice, human rights.

\section{Introducción}

En las dos últimas décadas, ha nacido un movimiento dinámico para mejorar la protección social y de salud entregada por profesionales. Una revisión rápida de "Google Scholar" en marzo de 2005 arroja que 8.600 artículos en inglés contenían las palabras 'evidence-based social' ('social basado en la evidencia') en el título: 'Trabajo Social basado en la evidencia'; 'protección so- cial basada en la evidencia'; 'servicios sociales basados en la evidencia'; política social basada en la evidencia'. En marzo de 2006, un estudio similar reveló un total de 1.500.000 artículos, libros y ponencias sobre 'Trabajo Social basado en la evidencia' y 'asistencia social basada en la evidencia', y 1.260.000 sobre 'política basada en la evidencia'. ¿Por qué ha aumentado tan masivamente el interés? ¿Se trata solamente de una nueva ideología o es más que eso?

* Ponencia presentada en el 33 Congreso Mundial de Trabajo Social IFSW/IASSW, Chile 2006. 
En este artículo se sostiene que el Trabajo Social y la política social basados en la evidencia son un imperativo de derechos humanos. Ya no es aceptable que al momento de formular políticas sociales los gobiernos o los agentes de Trabajo Social, como los trabajadores sociales, se involucren en la vida de las personas sin una evidencia que demuestre que lo que hacen puede ser beneficioso $-\mathrm{O}$ al menos no dañino- para quienes son el objetivo de la política o la intervención. La historia está llena de ejemplos de abusos de derechos humanos perpetrados por profesionales supuestamente "bondadosos", quienes han sometido a otras personas a experimentos deliberados. Este estudio sostiene que cuando los profesionales y legisladores intervienen en la vida de los demás sin una evidencia que muestre que lo que hacen podría ser beneficioso para los involucrados, no son menos culpables del daño causado y de abuso de derechos humanos simplemente porque pretendían hacer el bien. Este trabajo se divide en dos partes. La primera considera el caso del Trabajo Social y sus implicancias para la enseñanza de Trabajo Social, y la segunda considera la política social.

\section{Los principios de los Derechos Humanos son la esencia del Trabajo Social}

En este encuentro de trabajadores sociales y profesores de Trabajo Social, resulta apropiado que recordemos los objetivos del Trabajo Social y sus metodologías.

Resultó interesante leer el documento final de discusión que circuló sobre "Estándares globales para la educación y capacitación en Trabajo Social" (IASSW, 2004). Dadas las amplias diferencia internacionales en la práctica de Trabajo Social, debe haber sido un documento difícil de preparar. El escrito resume de manera excelente los estándares en cuanto a la diversidad cultural y étnica, además de la inclusividad de género. Los estándares acerca de currículos centrales también están bien presentados, y se enfatiza la importancia de un entendimiento cabal de deficiencias socio-estructurales: un conocimiento del comportamiento humano y de la transacción persona -en- ambiente y cómo las creencias y costumbres culturales influyen en el funcionamiento humano; conocimiento de profesiones afines para facilitar la colaboración interprofesional; conocimiento de políticas, servicios y leyes de bienestar social; la importancia de la defensa y empoderamiento de las personas, la identificación de fortalezas en las familias y una apreciación de la diversidad. Si bien los currículos recomendados son de utilidad para comprender estos temas, parecían deficientes en cuanto a lo que el trabajador social hace realmente "para promover el cambio social, la resolución de problemas en las relaciones humanas y el empoderamiento y la liberación del pueblo para incrementar el bienestar". Más preocupante resulta el hecho de que mientras el trabajador social tenga los valores correctos, hay muy poco que sugiera que él o ella realmente puede hacer daño. Si bien la definición de Trabajo Social indica que sus metodologías se basan en "un cuerpo sistemático de conocimientos sustentados en la experiencia y derivados tanto de la investigación y evaluación de la práctica", existe poca evidencia de esto en los currículos recomendados. Quizás deberíamos dudar de que podemos estar seguros de que los derechos humanos y la justicia social son realmente la esencia del Trabajo Social, mientras soltamos un ejército de trabajadores sociales en el mundo con el potencial de hacer un daño considerable (IASSW, 2004).

\section{Definición IFSW de Trabajo Social}

"La profesión de Trabajo Social promueve el cambio social, la resolución de problemas en las relaciones humanas y el fortalecimiento y la liberación del pueblo para incrementar el bienestar. Mediante la utilización de teorías sobre comportamiento humano y los sistemas sociales, el Trabajo Social interviene en los puntos en los que las personas interactúan con su entorno. Los principios de los Derechos Humanos y la Justicia Social son fundamentales para el Trabajo Social".

\section{Metodología}

“... El Trabajo Social basa su metodología en un cuerpo sistemático de conocimientos sustentados en la experiencia y derivados tanto de la investigación y evaluación de la práctica, incluyendo los contextos locales e indígenas" (IFSW, 2006). 


\section{Ejemplos de daño}

Para quienes nos recibimos en los años 70 , una lectura obligatoria era Medical Nemesis de Ivan Illich (Illich 1976). La esencia de su provocador mensaje era la nueva epidemia de lo que él llamó "iatrogénesis". "Iatrogénico" hacía referencia a un trastorno o enfermedad causada de manera involuntaria por el médico, debido a un diagnóstico o tratamiento incorrecto. Según Illich, "el médico se convierte en el agente patógeno". El libro contenía buenos argumentos y ejemplos excelentes. Cuarenta años más tarde, todavía lo tengo grabado en mi memoria. A pesar de que leí el libro Can Social Work Survive? de Brewer y Lait (1978), nadie sugirió que lo que yo haría podría empeorar la vida de mis pacientes. Recuerdo que cuando comencé la práctica, pensaba que se me había entregado mucha información acerca de cómo ocurrían los problemas sociales, pero muy poca sobre qué hacer con dichos problemas. Ahí estaba yo, con una licencia de Trabajo Social para experimentar con la vida de las personas.

Los ejemplos de daño abundan. Está la historia del estudio Cambridge-Somerville. En este estudio, un grupo de niños con un alto riesgo de convertirse en delincuentes se repartieron al azar entre la opción de no intervención y la opción de entrar a una estrategia planificada de apoyo social y psicológico. Treinta años después, se descubrió que la intervención había hecho una diferencia muy significativa en cuanto a criminalidad, alcoholismo, psicosis y muerte temprana. Aquellos que participaron en el programa de apoyo social habían tenido más problemas que quienes no participaron en el programa (McCord, 1992).

En otro ejemplo más práctico, muchos creen que un debriefing ${ }^{1}$ psicológico tras un incidente grave previene el trastorno por estrés postraumático (TEPT). Las investigaciones no respaldan esta creencia. Hasta ahora, los datos acerca de la efectividad del debriefing son abrumadoramente negativos, particularmente en evaluaciones de seguimiento. Por ejemplo, según Carlier et al. (1998), entre los policías que acudieron a un accidente aéreo, aquellos que se sometieron a un debriefing mostraron un número significativamente mayor de síntomas de hiper-alerta relativos al desastre en un seguimiento 18 meses después que quienes no recibieron el tratamiento. De acuerdo a Mayou et al. (2000), entre las personas que acudieron a un hospital tras un accidente de tránsito, aquellas que recibieron $\mathrm{CISD}^{2}$ tenían un resultado significativamente peor en tres años, en términos de síntomas psiquiátricos generales, ansiedad de viaje y nivel general de funcionamiento. Según Bisson et al. (1993), entre una muestra de víctimas de trauma de quemadura un $26 \%$ del grupo de debriefing tuvo TEPT en el seguimiento tras 13 meses, comparado con un 9\% del grupo control. Es importante señalar que de acuerdo al Informe Cochrane de 11 ensayos clínicos no se encontró evidencia de que el debriefing redujera la morbilidad psicológica general, depresión o ansiedad, y se recomendaba la suspensión del debriefing obligatorio para víctimas de trauma (Rose, et al. 2001). Sin embargo, el debriefing de incidentes graves continúa siendo una terapia generalizada, a pesar de la evidencia que muestra que hace daño. En un área más parecida a la política social, otro ejemplo es la emigración de niños y niñas sin acompañamiento a Canadá y Australia. Hasta la década de los 60 y de manera bien intencionada, Barnardo's - una respetada ONG del Reino Unido- envió a niños y niñas sin acompañamiento desde sus hogares residenciales, en el Reino Unido a una nueva vida en Canadá. Muchos padres no estaban del todo conscientes de lo que ocurría con sus hijos e hijas. Se estima que Barnardo's llevó más de 20.000 niños y niñas a Canadá (Barnardo’s 2005). De manera similar, entre 1947 y 1953 se recibieron en Australia más de 3.200 niños y niñas desde el Reino Unido y Malta. En muchos de estos proyectos, los niños y niñas fueron ubicados en hogares dirigidos por instituciones religiosas y grupos de protección social como Christian Brothers, Barnardo's y el movimiento Fairbridge (National Archives of Australia 2006).

Si bien a muchos de estos niños les ha ido bien, otros recibieron un cuidado deficiente y/o sufrieron de negligencia o abuso, y muchos dicen hoy estar traumatizados por la experiencia de haberse separado de sus familias y no tener un sentido de identidad. En esa época se creía que la emigración les daría a los niños un maravilloso nuevo comienzo en un mundo nuevo. En 2004 los representantes de estos niños emprendieron acciones legales en contra de Barnardo's por el daño que habían sufrido (Barnardo's 2005). Existen muchos otros ejemplos,

1 Análisis, revisión (N. E).

2 Critical Incident Stress Debriefing (CISD), o revisión de un incidente crítico de stress, es un tipo de intervención basada en una sesión grupal única, para víctimas expuestas a un evento traumático usado para prevenir post traumatic stress disorder. (N.E.). 
pero todos refuerzan el hecho de que los trabajadores sociales deben saber más acerca de la práctica basada en la evidencia si no quieren convertirse en "el agente patógeno".

\section{¿Qué es el Trabajo Social basado en la evidencia?}

Definición de Trabajo Social basado en la evidencia:

"Es la integración de la mejor evidencia científica con experiencia clínica y valores" (Sackett, Straus, Richardson, Rosenberg \& Haynes, 2000 pl)

"Es el uso conciente, explícito y juicioso de la mejor evidencia actual en la toma de decisiones sobre la atención de pacientes individuales" (Sackett et al, 1997, p2)

"La atención de salud basada en la evidencia se refiere al uso de los mejores conocimientos actuales en la toma de decisiones sobre grupos y poblaciones" (Gray 2001)

En la primera definición se destacan los tres componentes: mejor evidencia científica, experiencia clínica y valores. Los valores no sólo se relacionan con comprender la diversidad y los valores culturales. Se sostiene que es éticamente inaceptable experimentar con la vida de las personas a menos de que exista alguna evidencia que indique que lo que se hace en el nombre del bien realmente puede ser de ayuda.

Implementar enfoques basados en la evidencia es un gran desafío. Implica un cambio de actitud y humildad para aceptar nuevos perspectivas. Una de las mayores barreras en la práctica basada en la evidencia es la tradición: "siempre hemos hecho las cosas de esta manera". Otra es: "a las personas les gusta lo que hacemos". Por muy bien intencionadas que sean, las costumbres más tradicionales pueden hacer daño, como lo demuestran numerosos ejemplos en todo el mundo.

\section{¿Qué debe hacerse antes de llegar a las intervenciones basadas en la evidencia?}

Hay cinco etapas en el desarrollo de la práctica basada en la evidencia.

El punto de partida es la identificación de un comportamiento o una condición como problema social. Al decidir qué es un problema social, deberá contextualizarse de acuerdo a las normas y valores, ética y moralidad, y la identidad grupal/nacional en una sociedad en particular. Distintas sociedades en épocas diferentes tendrán visiones distintas acerca de lo que es o lo que no es un problema social (Buchanan, 2000).

Cuando se ha definido un problema social, las siguientes dos etapas son descubrir el alcance del problema social (o epidemiología) y lo que se cree son las causas (etiología). A nivel de Trabajo Social, en un área local se pude querer establecer un proyecto para prevenir que los jóvenes cometan delitos. En este caso, se recopilará información acerca de la cantidad de delitos, el número de delincuentes juveniles conocidos y las características de los delincuentes juveniles, y se investigará la literatura internacional para ver si los delincuentes juveniles de occidente compartían características similares. De ser así, podría ser adecuado "replicar" una terapia "efectiva" de los Estados Unidos y adaptarla a las necesidades locales. En los Estados Unidos se ha invertido en numerosos Ensayos Clínicos Aleatorizados y Controlados (ECAC). Al igual que en la medicina, los pacientes -o en este caso los delincuentes juveniles- se reparten de manera aleatoria en dos tipos de tratamiento. Al final del tratamiento,

FIGURA 1

ETAPAS PARA LA PRÁCTICA BASADA EN LA EVIDENCIA (de Buchanan et al 2000)

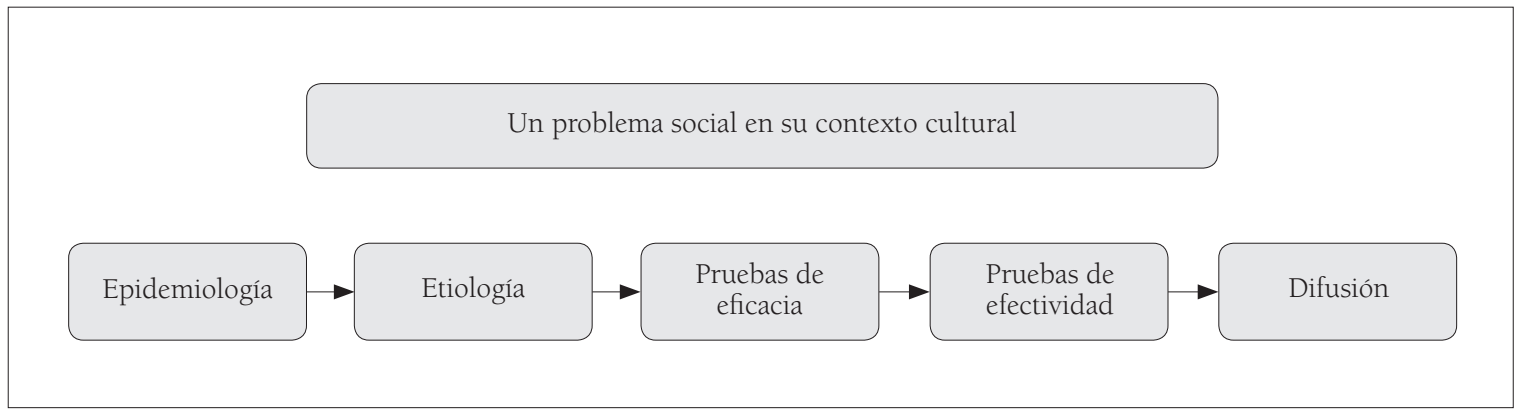


se ve a qué grupo le ha ido mejor. Estos ensayos se llaman Pruebas de Eficacia y suelen llevarse a cabo en centros de excelencia. En este caso se procede a las Pruebas de Efectividad, lo que implica poner a prueba el programa modelo en un entorno no clínico. ¿Funciona este modelo de tratamiento en nuestro entorno? La etapa final es la difusión, donde se replica un programa efectivo en un país o área que tenga características similares (Buchanan, 2000). En la actualidad, muchos programas ofrecen capacitación a los profesionales para garantizar "fidelidad", es decir, que el programa se lleve a cabo según lo esperado.

Existen otros problemas sociales que pueden ser más complejos. Por ejemplo, el VIH/SIDA tiene una etiología muy diferente en distintas partes del mundo. En los Estados Unidos se trata de un problema que afecta principalmente a la comunidad homosexual, en África es un problema heterosexual y en algunos lugares de Asia es un problema que afecta a las mujeres afectadas por el comercio sexual. Por lo tanto, no tendría sentido "replicar" un programa de Estados Unidos que podría tener como objetivo un sector equivocado de la población. Antes debe llevarse a cabo un análisis de necesidades o buscarse más información acerca de la epidemiología y la etiología (los factores de riesgo y protección).

Existe mucho debate acerca de los estándares de evidencia. Se necesita una evidencia científica sólida para evitar conclusiones, resultados o resultados esperados inválidos. Generalmente se aceptan los Ensayos
Clínicos Aleatorizados y Controlados (ECAC) como el "patrón oro" en las investigaciones. Sin embargo, muchas intervenciones de Trabajo Social involucran a poblaciones, escuelas y comunidades enteras donde puede ser más difícil y costoso organizar ECAC. Acá pueden ser muy útiles los datos estadísticos. Otros diseños, como los cuasi-experimentales y los estudios cualitativos donde el consumidor comparte sus experiencias, también pueden ser de real utilidad. Quizás la evidencia más sólida venga de diversos estudios de investigación utilizando distintas metodologías en diferentes contextos. Al final, el trabajador social debe tomar una decisión en base a las investigaciones disponibles y en el contexto en el que trabaja, con respecto a qué intervenciones tienen las mejores probabilidades de ser útiles y las menores probabilidades de causar daño.

\section{El profesional competente - Trabajo Social basado en la evidencia}

Eileen Gambrill (2005) cree que cuando se trabaja a nivel del individuo, la evidencia científica es solo una parte del todo:

Primero el profesional debe comprender las características del paciente y las circunstancias en las que vive él/ella o la familia. En este sentido, los trabajadores sociales deben conocer tanto el alcance del problema como sus posibles causas. Luego, el profesional competente debe establecer las preferencias y deseos del paciente. En un mundo ideal

FIGURA 2

UN MODELO PARA LAS DECISIONES BASADAS EN LA EVIDENCIA EN LA PROTECCIÓN SOCIAL (Gambrill, 2005)

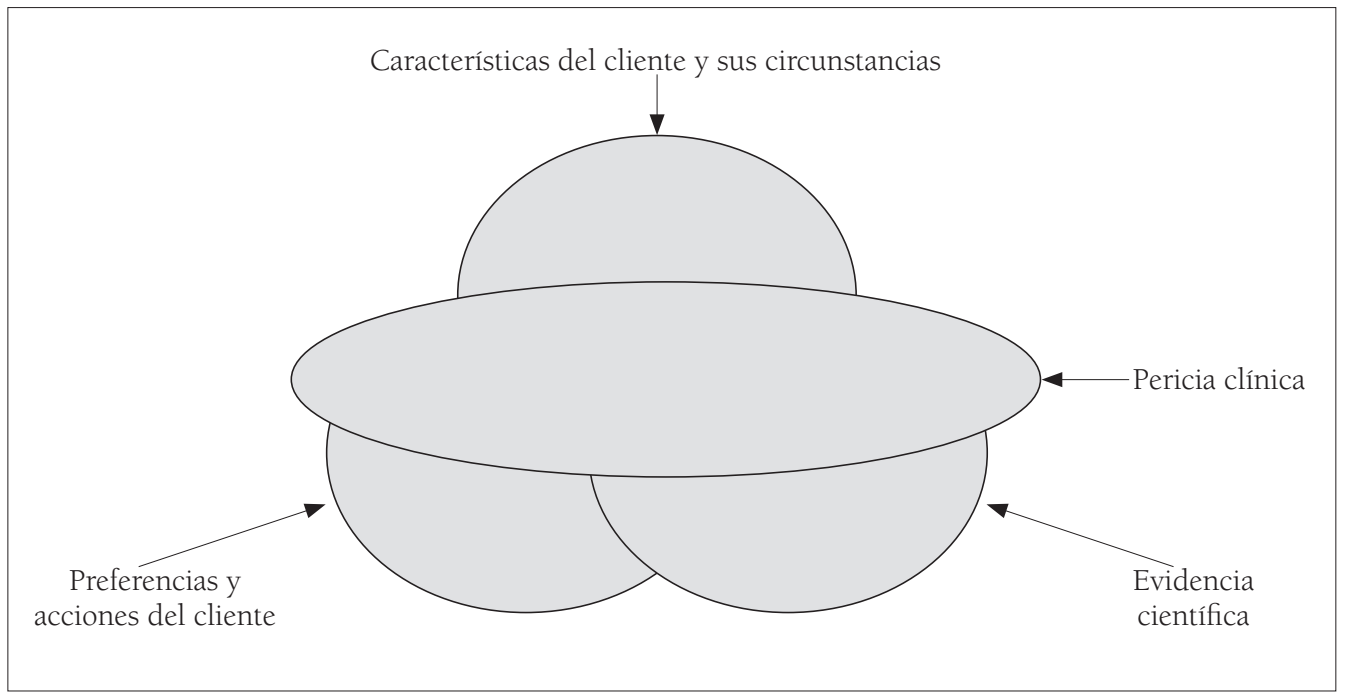


se podría preguntar al paciente: "¿cuál sería su escenario ideal?" Luego, este "escenario ideal" debe ajustarse a las realidades actuales de la situación de cada paciente. Por ejemplo, en casos de delincuencia, maltrato infantil y salud mental, esto puede significar asegurarse de que el paciente comprenda las repercusiones legales de sus actos. Posteriormente, el profesional aplica lo que conoce en base a la evidencia científica. Por ejemplo, en una situación de maltrato infantil donde el padre es muy agresivo, puede haber un programa local para el control de la ira que podría ayudar. Pero esto no es todo. Cada persona es única. Al final existe un elemento de experiencia clínica al decidir cuáles pueden ser las mejores intervenciones para ayudar al paciente.

\section{Oxford - un centro de actividad basada en la evidencia}

En el Reino Unido, Oxford ha estado en el centro de gran parte de este movimiento. En 1993 Sir Ian Chalmers fundó la Cochrane Collaboration. Sir Ian era un médico general que sentía que los médicos se confundían con la cantidad de investigaciones y los distintos tratamientos recomendados para los mismos síntomas. El juramento hipocrático "no hacer daño" sustenta la práctica médica en todo el mundo, pero ¿cómo podría un médico general moderno asegurarse de que su práctica se basa en "la mejor evidencia" de que está ayudando a no dañar al paciente?

Ian Chalmers refinó la metodología de las "revisiones sistemáticas". Se trata de revisiones que analizan sistemáticamente los hallazgos de diversos estudios de investigación acerca de un tema en particular y llegan a una conclusión general acerca de los méritos de tratamientos específicos para problemas específicos. Actualmente la Cochrane Collaboration es una organización internacional e independiente sin fines de lucro, dedicada a elaborar información actualizada y precisa sobre los efectos de la atención médica disponible en el mundo. Produce y circula revisiones sistemáticas de intervenciones de atención médica y promueve la búsqueda de evidencia en la forma de ensayos clínicos y otros estudios de intervenciones. El principal producto de la Collaboration es la Cochrane Database of Systematic Reviews [Base de Datos Cochrane de Revisiones Sistemáticas], que se recopilan en The Cochrane Library (Cochrane, 2006) y que están disponibles en Internet sin costo. Muchos de nuestros estudiantes utilizan revisiones publicadas por Cochrane Libray para sus tesis de Magíster.

\section{La Campbell Collaboration - la división de educación, bienestar social y justicia penal}

El siguiente paso lógico fue la idea de que las investigaciones sobre intervenciones sociales también debían revisarse de manera más sistemática. En diciembre de 2000, el Centro Cochrane en el Reino Unido transfirió los elementos de educación, bienestar social y justicia penal de su trabajo a una nueva organización, la Campbell Collaboration. Esta organización comenzó a aumentar el archivo de inmediato. Hoy existen casi 12.000 ensayos aleatorios y posiblemente aleatorios sobre educación, bienestar social y justicia penal. Cada registro en el archivo contiene información sobre citaciones y disponibilidad, y por lo general incluye un resumen. El público puede acceder a ellos sin costo a través de Internet.

\section{Magister en Trabajo Social basado en la evi- dencia de la Universidad de Oxford}

La Barnett House, en la Universidad de Oxford, tiene una larga trayectoria en investigaciones sobre problemas sociales, enseñanza y práctica. Como tal, quizás fue la precursora del actual movimiento basado en la evidencia. Se estableció en 1914 en memoria de Canon Barnett, un antiguo rector de Toynbee Hall, la primera residencia universitaria que se había fundado en Whitechapel 30 años antes para dedicarse a los ideales del servicio social e investigación social. La nueva institución en Oxford sería un centro para el estudio de problemas sociales y económicos, además de la educación y preparación de hombres y mujeres para el Trabajo Social y la investigación social.

En años recientes, ha habido dos corrientes interrelacionadas en el Departamento. Por un lado, existe un Programa de Magíster en Política Social Comparativa, y por otro está el Magíster en Trabajo Social Profesional. En los diversos centros de investigación se realizan importantes programas de investigación, particularmente: el Centro de Investigación Basado en la Evidencia, del cual soy miembro, el Centro de Investigación sobre Desventaja Social, el Centro Oxford para el Derecho de Familia; mi propio centro, el Centro para la Investigación de Paternidad e Hijos y el Proyecto Oxford de Población (OXPOP). En el año 2003, en un emocionante nuevo desarrollo, nació el Magíster en Trabajo Social Basado en la Evidencia. Con él se reemplazaba el programa de capacitación profesional anterior para trabajadores sociales, y se expandía la corriente doctoral (www. apsoc.ox.ac.uk). Recientemente los gobiernos del Reino Unido, Suecia, Noruega y otros han solici- 
tado al Departamento que realice un número de revisiones sistemáticas para informar sobre políticas y prácticas futuras. Importantes ensayos de intervención también dicen relación con intervenciones de paternidad, divorcio y una importante nueva corriente liderada por Don Operario sobre intervenciones sociales para el VIH/SIDA.

\section{Entonces, ¿qué hemos aprendido?}

Los últimos tres años han sido una rápida curva de aprendizaje. Nuestros estudiantes vienen de todos los continentes en el mundo: Chile, Argentina (todavía no Brasil), los Estados Unidos, Canadá, países europeos post-comunistas, Rusia, India, China y Australasia. Al comenzar el curso nos propusimos encontrar "intervenciones basadas en la evidencia". Los estudiantes utilizaron Internet para encontrar evidencia que apoyara las intervenciones para condiciones como la depresión posparto, el VIH y el abuso de drogas. Recuerdo muy bien el primer año. En el grupo de depresión, el alumno de los Estados Unidos encontró diversos ECAC que mostraban que la Terapia Cognitivo-Conductual individual era efectiva para tratar a mujeres con depresión. El alumno de Rumania señaló que "era espléndido, pero en Rumania había un trabajador social por al menos cada 1.000 mujeres con depresión, y no había cómo organizar un tratamiento individual". El alumno de India dijo que él también tenía un problema, ya que "en India no existe la palabra depresión". Los estudiantes continuaron la discusión. No se trataba de que en India no hubiese madres con depresión. La tasa de suicidios de madres lo desmentía, pero era un tema sensible. Entonces, ¿cómo podían ayudar a estas madres sin causarles un estigma potencialmente dañino? Finalmente los estudiantes decidieron que, ya que la mayoría de las madres asistían a algún tipo de clínica de servicio social para niños y niñas, era necesario capacitar a los trabajadores para identificar y obtener un apoyo discreto para las madres con depresión.

La enseñanza sobre VIH ha sido igualmente dramática. Actualmente Don Operario trabaja en una investigación sobre intervenciones sociales para reducir la frecuencia de VIH/SIDA en China. En ese país la homosexualidad es ilegal, por lo que el VIH/ SIDA contraído mediante ese tipo de relaciones debe manejarse con especial sensibilidad. Muchas personas también se han infectado al comprar sangre que venden las personas pobres de las áreas rurales, algunas de las cuales están infectadas con VIH. Lo que Don debe hacer es comprender la naturaleza de las sensibilidades del problema antes de que pueda realizarse alguna intervención. El potencial de hacer más daño es muy alto.

Tal vez lo más importante es que hemos aprendido que existe una jerarquía de evidencias. Si bien el Ensayo Clínico Aleatorizado y Controlado puede ser el patrón oro, este mismo tiene sus limitaciones, ya que solo hace referencia a una muestra en particular, en un momento en particular, y puede no ser culturalmente apropiado. Ahora sabemos que la mejor evidencia tiene múltiples focos que vienen de distintas investigaciones, y que ha utilizado metodologías y muestras diferentes en distintas partes del mundo. Lo que hemos debido hacer es entrenar a nuestros estudiantes para que sean críticos con respecto a la investigación y que comprendan lo que las investigaciones pueden y no pueden decir.

Principalmente, hemos aprendido que quienes intervienen en la vida de los demás, particularmente como agentes del estado, sin importar en qué parte del mundo trabajen, necesitan estar conscientes de que pueden hacer daño. El hecho de que no existan investigaciones disponibles en su área o en su tema no es excusa. El trabajador social debe descubrir lo que se sabe de otras áreas, juzgar cuán relevantes pueden ser los hallazgos para su trabajo y luego decidir si una intervención efectiva conocida de otra área debe adaptarse a las necesidades de sus pacientes. Además, debe asegurarse de evaluar lo que está haciendo e informar a otros sobre sus hallazgos. Es así como construimos el banco del conocimiento.

\section{Política social basada en la evidencia}

En cuanto a la política social, la tarea es más difícil. La política basada en la evidencia todavía se encuentra en una etapa embrionaria. En muchas sociedades, las fuerzas políticas dominantes son fuertemente ideológicas, y estos gobiernos están menos interesados en utilizar las investigaciones para informar acerca de políticas. A John Milton Keynes se le atribuye el haber dicho: "no hay nada que los gobiernos odien más que estar bien informados, porque hace que los procesos de tomar decisiones sean mucho más complicados y difíciles". También se dice que Ronald Reagan tomó la mayoría de sus decisiones basado en la astrología, iy la verdad es que le sirvió bastante! (Mulgan, 2006). Pero los tiempos están cambiando.

Actualmente, las instituciones globales, la OCDE, el Banco Mundial, las reformas de mercado europeas y el PNUD están haciendo estudios comparativos for- 
males acerca de cuán bien les va a los distintos países (por ejemplo, el Informe de las Naciones Unidas sobre el Desarrollo, 2004). Una de las fuerzas para el cambio más potentes del gobierno británico ha sido aprender cuán bien les ha ido comparado con otros países. Esto demostró que el Reino Unido tendía a compararse con los Estados Unidos, pero a los Estados Unidos por lo general les iba mucho peor que al Reino Unido en temas como el crimen, la política social y los niños y niñas viviendo en la pobreza, mientras que a otros países en Europa les estaba yendo mucho mejor. La globalización del conocimiento hace difícil esconder los resultados de malas políticas de gobierno. Los medios están en todos lados, demostrando distintos estándares de vida, discriminación y abusos de derechos humanos. Con Google, en 0,14 segundos cualquier ciudadano puede reunir un conjunto creíble de información sobre cualquier asunto para el cual desee buscar una respuesta. Pero ¿hasta qué punto podemos discutir que un mal gobierno - o un gobierno que ignora la evidencia para implementar mejores políticas - está cometiendo abusos de derechos humanos? La Enciclopedia Filosófica Stanford ( 0,84 segundos en Google) resume los derechos inherentes en la Declaración Universal de Derechos Humanos, 1948.

\section{Declaración Universal de Derechos Humanos (Naciones Unidas 1948)}

a. Derechos de seguridad - protección contra el asesinato, masacre, tortura y violación.

b.Derechos de libertad: protección de la libertad en áreas como creencia, expresión, asociación, reunión y movimiento.

c. Derechos políticos: reuniones, protestas, votaciones, servicios en cargos públicos.

d.Derechos al debido proceso que protegen contra los abusos del sistema legal, como encarcelamiento sin juicio, juicios secretos y castigos excesivos.

e. Derechos de igualdad; ciudadanía igualitaria, igualdad ante la ley.

f. Derechos de bienestar (o derechos económicos y sociales): provisión de educación, protecciones contra propiedad y hambre.

Cualquier país que ratifica un acuerdo de derechos humanos de las Naciones Unidas se compromete a respetar e implementar los derechos cubiertos por el acuerdo. También se compromete a aceptar y responder a un escrutinio internacional y crítica de su cumplimiento. El PIDCP señala que los estados participantes deben informar periódicamente acerca de su cumplimiento del acuerdo. "Los derechos humanos son una aspiración... se trata de evitar lo terrible en lugar de lograr los mejores estándares mínimos" (Stanford Encyclopedia, 2006). El dilema es que en un mundo globalizado los estándares están cambiando y que lo "terrible" y lo "mínimo" son objetivos en movimiento. ¿Deberían los ciudadanos estar felices con políticas ineficaces para aliviar la pobreza y mejorar la educación y la salud cuando las investigaciones pueden demostrar que hay mejores maneras de alcanzar los mismos fines por un costo similar? Los gobiernos democráticos pueden estar impulsados por las ideologías y ser bien intencionados, pero ¿no es un abuso de los derechos humanos negar remedios eficaces a los ciudadanos para sus males sociales?

Por supuesto, los gobiernos tienen otras consideraciones. La evidencia o el conocimiento viene en muchas formas (Mulgan, 2006). Existe el conocimiento de política: por ejemplo, ¿qué funciona para combatir el crimen? Luego está el conocimiento científico: ¿qué es responsable del cambio climático? También está el conocimiento profesional, el conocimiento que viene de enseñar acerca de la práctica médica o de bienestar social. Algo muy importante en las sociedades democráticas es el conocimiento de la opinión pública, que puede o no coincidir con el conocimiento científico. El desafío para el servicio público en cualquier país es cómo entender todos estos distintos tipos de conocimiento.

Otra dificultad es que en algunas áreas existe un amplio consenso acerca de lo que se sabe, por ejemplo, microeconomía, mercados laborales, algunas áreas de la medicina - acá el conocimiento de política es similar a la ciencia natural. Pero en otras áreas existe un gran desacuerdo sobre lo que se sabe, y la evidencia es muy delgada. En estos campos, los gobiernos democráticos tal vez deban adelantarse a las investigaciones para responder a los requerimientos de sus constituyentes, pero siempre estará disponible una fuente de conocimiento bastante confiable. Cuando falta conocimiento, quienes experimentan los males sociales pueden ser informantes confiables sobre el impacto de las políticas de gobierno.

\section{¿Cómo puede la política basarse en la evidencia?}

En el Reino Unido, recientemente concluí una revisión sobre el impacto de políticas gubernamentales en niños y familias en riesgo de exclusión social (Buchanan et al. 2004). Esto puso en relieve las 
muchas maneras -aunque aun son imperfectas- en que el Reino Unido de Blair intenta incorporar la evidencia a los procesos de elaboración de políticas. En la etapa de formación de políticas, grupos interesados claves realizan sondeos, generalmente en focus groups en los que participan académicos, y en las mismas condiciones con usuarios y proveedores de servicios. Le sigue un proceso más amplio de consulta, tal vez en la forma de un Libro Verde. Una vez que se formula una política el gobierno publica Acuerdos de Servicio Público (PSA, por sus siglas en inglés), que señalan los objetivos de la política en un área en particular, por ejemplo para aumentar logros educativos entre los jóvenes. Para monitorear la política, los documentos gubernamentales describen indicadores de desempeño, que mostrarán si la política va por buen camino. Estos serán, por ejemplo, los niveles que han logrado niños que viven en la pobreza en las Pruebas de Rendimiento Estandarizado (SAT, por sus siglas en inglés) a los 7,11 y 14 años en diversos colegios. Otro indicador es la cantidad de ausentismos escolares en un período en particular. Se publican objetivos acerca de lo que el gobierno espera lograr mediante sus políticas: por ejemplo, para aumentar el número de niños que reciben comidas gratis o que alcanzan el nivel 4 en las SAT, o para reducir el número de días educativos perdidos por ausentismos injustificados. Luego se entregan fondos a las organizaciones para enfrentar bajos niveles de desempeño y altos niveles de ausentismo

Para evaluar la política, el gobierno tiene una variedad de datos potenciales. Tiene los "datos de tendencia" o las estadísticas de los indicadores de desempeño recopilados por estadísticos gubernamentales. En muchos casos estos se publican para "nombrar y avergonzar", por ejemplo, autoridades educacionales con bajo desempeño. El Gobierno también solicita evaluaciones de distintos aspectos de sus políticas a evaluadores externos. Para nuestro trabajo para la Unidad de Exclusión Social, se nos pidió que hiciéramos una revisión de todas las evaluaciones y de la literatura formal y "gris". Finalmente, para este proyecto también solicitaron estudios de caso: entrevistas cualitativas en profundidad con padres e hijos que podrían verse afectados por el problema.

En los Proyectos de las Naciones Unidas para el Desarrollo se utiliza un enfoque muy similar. Por ejemplo, los Objetivos de Desarrollo del Milenio (ODM) representan un conjunto de metas y objetivos mensurables y sujetos a limitaciones de tiempo para combatir la pobreza, el hambre, las enfermedades, el analfabetismo, la degradación medioambiental y la discriminación hacia las mujeres. Surgidos de la Cumbre del Milenio de las Naciones Unidas de 2000, los objetivos son el medio para acelerar el paso del desarrollo en el mundo y para medir resultados concretos (UNDP 2004). En un mundo globalizado es difícil ignorar la evidencia, en especial cuando hay sólidas investigaciones sobre cómo se puede mejorar la protección y el bienestar de los ciudadanos, respetando costumbres culturales y reconociendo la disponibilidad de recursos económicos. En este trabajo diríamos que hacer eso -ignorar la evidencia- sería un abuso de derechos humanos.

\section{Trabajo Social y política social basados en la evidencia. ¿Una nueva ideología o un imperativo de Derechos Humanos?}

El viaje recién ha comenzado. En el Trabajo Social y en la educación de trabajadores sociales tenemos un largo camino que seguir antes de que podamos decir que nuestra práctica está "basada en la evidencia”. Cuando comencé mi práctica como trabajadora social, un sabio psiquiatra mayor me dijo en 1970: "en mi vida, no hemos sabido realmente lo que les haremos a las personas, pero en tu vida podrían saberlo". El banco de conocimientos en el Trabajo Social está creciendo. La política social basada en la evidencia tiene tal vez un viaje mucho más largo. Recién estamos comenzando a saber cómo hacer un mejor uso del conocimiento que está disponible. Sin embargo, insisto en que la política y práctica basadas en la evidencia no son solo una nueva ideología; son un imperativo de derechos humanos. El solo hecho de que tengamos buenas intenciones no nos hace menos culpables del daño hecho a los demás que si nuestros actos fuesen deliberados.

\section{Referencias}

BARNARDO'S. (2005). Immigrant children in Canada. www.barnardos.org.uk/whatwedo/aftercare/canada. jsp.

BISSON, J.I., JENKINS P. L., ALEXANDER J., BANNISTER C. (1993). Randomized controlled trial of psychological debriefing for victims of acute burn trauma. British Journal of Psychiatry; 171:78-81.

BREWER, C. Y. \& LAIT, J. (1978). Can Social Work Survive? Temple Smith, Londres.

BUCHANAN, A. (2000). Present Issues and Concerns in A. Buchanan and B. Hudson: Promoting Children's Emotional Well-being. Oxford: OUP 
BUCHANAN, A., BENNETT, F., SMITH G., SMITH T., RITCHIE, C. AND VITALI, S. (2004). The Impact of Government Policy on Children aged 0-13 and their families at risk of social Exclusion. Londres: Social Exclusion Unit. www.socialexclusion.gov.uk/downloaddoc. asp?id=266.

CAMPBELL COLLABORATION: www.campbellcollaboration.org/Fralibrary.html

CARLIER, I.V.E., LAMBERTS, R.D., VAN UCHELENNH, A.J. (1998). Traumatic stress in police officers: a field study of the impact of debriefing. Stress Medicine: $14: 143-148$.

COCHRANE. (2006). www.cochrane.org/docs/newcomersguide

GAMBRILL, E. (2005). Evidence-based Social Work. Talk to the Faculty of Social Studies, University of Oxford. Febrero 21

GRAY, J.A.M. (2001). Evidence-based healthcare. NY: Churchill Livingstone

IASSW. (2004). www.iassw-aiets.org/en/About_IASSW/ GlobalStandards.pdf

IFSW. (2006). Definition of Social Work IFSW, www.ifsw. org/en/p38000208.html.

ILLICH, IVAN. (1976). Medical Nemesis : the expropriation of health. Nueva York: Pantheon Books.

MAYOU RA, EHLERS A., HOBBS M. (2000). Psychological debriefing for road traffic accident victims. British Journal of Psychiatry; 176:589-593.
MCCORD, J. (1992). The Cambridge-Somerville study: a pioneering longitudinal experimental study of delinquency prevention. En McCord, J. Temblay, R. E., (eds.) Preventing antisocial behavior: interventions from birth through adolescence. Guildford Press, Nueva York.

MUlGAN, G. (2006). The Academic and the PolicyMaker. Talk at the launch of the Public Policy Unit, University of Oxford. Febrero, 2006 Oxford: Department of Politics and International Relations.

NATIONAL ARCHIVES OF AUSTRALIA. (2006). www.naa.gov.au/publications/fact_sheets/fs185.html

ROSE S., BISSON J., WESSELY S. (2001). Psychological debriefing for preventing post traumatic stress disorder (PTSD). Cochrane Database Systematic Review; 3.

SACKETT, D. L., RICHARDSON, W. S., ROSENBERG, W., et al (1997). Evidence-based Medicine. Edimburgo: Churchill Livingstone.

SACKETT, D. L., STRAUS, S. E., RICHARDSON, W. S., ROSENBERG, W. \& HAYNES, R. B. (2000). Evidence-based medicine: How to practice and teach EBM. Edimburgo: Churchill Living-stone.

STANFORD ENCYCLOPEDIA. (2006). http://plato. stanford.edu/entries/rights-human/

UNITED NATIONS. (1948) Universal Declaration of Human Rights UDHR United Nations (1948b) http:// plato.stanford.edu/entries/rights-human/

UNITED NATIONS DEVELOPMENT PROGRAMME. (UNDP) (2004). the National Millennium Development Goals Report, www.undp.hr/en/goals/goals_index. html 